\title{
On the Basis of Friendship - A Reply to Phelan
}

\begin{abstract}
What is common to all instances of friendship? Given their seemingly heterogeneous character, Phelan (2019) suggests that friendships are relationships that result from collaborative norm-manipulation. In this paper I suggest that this proposal fails to account for all friendships without relying on the notion of some kind of care.
\end{abstract}

Keywords: friendship, norms, care 


\section{On the Basis of Friendship - A Reply to Phelan}

Philosophical discussions of friendship have long revolved around particularly significant relationships involving mutual caring. In a recent paper, Phelan (2019) suggests that this focus has been misguided, claiming that it obscures many instances of genuine friendship. To right this error, he suggests that we should rather see 'collaborative norm-manipulation' as basic to and necessary for friendship. I will argue that this proposal faces problems when we consider which norms are the basic norms that friends manipulate. Either the basic norms are those that apply to particular social positions, in which case the account is too restrictive as to what counts as friendship. Or the basic norms are those that apply to strangers, in which case the account must rely heavily on a second condition of friendship, the 'mutual positive disposition' condition. However, this condition undermines the thought that norm manipulation might be a basic feature of friendship, since it is well placed to explain norm manipulation. Moreover, taking norm manipulation to be basic gets the order of explanation the wrong way around: friends manipulate norms because they are friends, not vice versa. I argue that Phelan therefore fails to provide a genuine alternative to the standard focus on deep relationships based on mutual caring.

Philosophical discussions of friendship frequently take their cue from Aristotle's division of friendships into three kinds: those of virtue, those of pleasure and those of utility. True friendships, he maintains, are the first kind: friendships of virtue. Whatever they have made of the particulars of his account, philosophers have generally agreed with the spirit of this remark and thought that true friendship is that in which the friends care about each other for their own sake, rather than for anything they might gain from each other, such as pleasure or utility. As such, when discussing friendship philosophers have generally focused on deep and important relationships that involve some significant degree of intimacy, mutual care, or love. ${ }^{1}$ Such philosophers generally distinguish mere acquaintances and those with whom we share activities from true friends, and thinking about friendship has been largely based upon the latter category.

Phelan suggests that this approach to thinking about friendship is wrongheaded. The initial reason he gives for preferring a more inclusive approach is derived from linguistic considerations: the term 'friendship', he notes, can be used seemingly univocally of both those we love and those with whom we have more distant relationships. He writes:

Many of the relationships that we refer to as friendships do not involve deep, mutual caring or lack the sort of intimacy that has been attributed to 'true' friendships. (Phelan 2019; 4)

In this vein, he notes that we would describe as 'friends' work colleagues who eat lunch together a couple of times a week but rarely interact otherwise, or those who meet up only to participate in a specific activity such as playing tennis together, even where such relationships do not involve mutual love or care (Phelan 2019; 3). He suggests that a philosophical account of friendship should

\footnotetext{
${ }^{1}$ For a selection of such cases, see Annis (1987), who describes friendship as a 'special bond' involving care (Annis 1987; 352); Thomas (1987), who describes 'deep friends' as 'loving' one another; Nehamas (2010), who assumes that we love our friends (Nehamas 2010; 278), and Helm (2010), who takes friendship as the paradigm of love.
} 
cover all such cases: unless there is a good explanation as to why some of these relationships should be ruled out, an account of friendship should cover all ordinary uses of the term.

If we therefore set aside features such as intimacy, love, or care, how might we instead understand friendship? Phelan focuses on the fact that within friendships our expectations and obligations often differ significantly from the expectations and obligations that we have within other relationships. The norms guiding our behaviour regarding our friends, that is, often differ significantly from those that guide our behaviour in other contexts. Phelan thus suggests that friendships are those relationships that are the result of participants' collaborative manipulation of norms. He writes:

All the diverse relationships we call friendships share at least one feature: they are all the product of significant collaborative norm manipulation and they consist in collaboratively agreed upon normative frameworks. (Phelan 2019; 6)

There are thus two conditions here. First, that friendship involves the manipulation of norms and, second, that such manipulation occurs collaboratively. This latter condition captures the idea that friendship is a symmetrical relationship; one of the potential friends cannot simply unilaterally decide to form a friendship. Rather, a friendship is formed through collaboration between two (or more) people.

To further illustrate his conception of friendship, Phelan provides some examples of the kind of norms that friends often manipulate. He notes that friends often manipulate norms concerning personal space, allowing for closer physical interaction than is otherwise socially permissible. Or they might manipulate norms concerning topics that can be broached in conversation, allowing for discussion of personal subjects that might otherwise be deemed intrusive (Phelan 2019; 7). He writes:

My tennis friend may make fun of my serve, a mere competitor should not. My drinking buddy may stand me a round, I will be inclined to buy another to repay a mere acquaintance. (Phelan 2019; 9)

In any of these cases, he regards it as important that the new norms introduced when one individual transgresses a background norm are subject to veto by the other. It is in this sense that he regards the adoption of the new norms as collaborative. For example, in first teasing one about one's serve, a friend would be attentive to one's reaction, standing ready to retract the transgression of the norm if one were hurt or offended.

Whilst Phelan maintains that the norm manipulation condition alone is not sufficient for friendship, which requires also a 'mutual positive disposition' (short of love or care), he nonetheless takes it to be not only a necessary feature of friendship, but also a basic feature of it. He thus suggests that norm manipulation is not merely incidental to friendship, but that it is an essential part of what makes two people friends. Friendship, on his account, consists in collaboratively agreed upon normative frameworks. As such, he takes it to be a feature that casts light on the nature of friendship itself. ${ }^{2}$

Whilst Phelan seems right that the norms which apply to friends are usually different to those that apply to mere strangers, I want to raise two problems with making norm-manipulation central to an account of friendship. Depending on which norms we take to be the basic set, the

2 Phelan states that this account "explains some key features of the friendship relation" and "is of explanatory value" (Phelan 2019; 11). 
model is either too demanding or too permissive. The 'mutual positive disposition' condition could be invoked to keep the account from being too permissive. But once the mutual positive disposition condition is introduced, it seems to be doing all the explanatory work. I will thus suggest that even if norm-manipulation is a feature of all friendships, it may be that this is a merely incidental rather than basic feature of friendship. Indeed, it may be a consequence of the very kind of feature that Phelan initially rejects.

Some kinds of relationship seem to be well understood in terms of the particular norms of those roles. For instance, to be the legal guardian of a minor is to be in a position such that there are certain norms that apply to one, certain expectations and obligations that one has (that one must take care of the minor, for example). However, Phelan does not attempt to give a substantive account of what the particular norms for friendship are. Plausibly, there are no particular such norms-different friendships will simply involve different ones. Thus, for instance, while some friendships involve close physical interaction, others do not. And while playful teasing is an important part of some friendships, it has no place in other friendships. Phelan thus suggests simply that norms of friendship are those which result from collaborative norm-manipulation.

We still might ask, however, about the kind of norm he intends be manipulated in creating a friendship. Two possibilities suggest themselves. The first is that the norms that get manipulated are norms that apply to the individuals on the basis of some specific social position that they occupy in relation to one another (for example, the norms of work colleagues, neighbours, or cousins). And the second is that these norms are just norms that apply to any set of strangers one encounters within a given cultural context. ${ }^{3}$ Both of these possibilities, I want to suggest, lead to problems for the account.

\section{Interpretation 1: Manipulation of the Norms of Specific Social Positions}

Diverse social norms apply in diverse social contexts. In normal life, for instance, it would be unacceptable to talk at someone for half an hour, but this is perfectly acceptable in a university lecture. It would generally be unacceptable to delegate an arduous task to a complete stranger, but a boss in a workplace is allowed to delegate such tasks to his employees. Since our social interactions are frequently structured around such social positions, we might take the 'background' norms to be those that apply to the various social roles we occupy. On this interpretation, Phelan's claim would be that friends are those who do not simply abide by the norms that apply to their pre-existing social positions or roles. Rather, in friendships the norms are altered relative to the social positions that the individuals occupy. Thus, for example, while certain norms will apply to individuals who occupy the role of 'business partner' relative to one another-e.g., they will be expected to work together regularly, to financially trust one another in certain respects, and to plan for the future together-they might also manipulate these norms, and their doing so might partially constitute a friendship. They may, for instance, start spending time discussing their dating lives, and this might constitute starting a friendship.

On this interpretation, then given any social position that people occupy relative to one another, then at least some norms that usually apply given their social positions must be manipulated in

\footnotetext{
3 The latter reading is perhaps suggested by Phelan's comment that '[i]t is against the backdrop of a cultural normative framework that strangers can become friends. And they will become friends through the collaborative manipulation of the shared norms which coordinated their interactions as strangers within a culture' (Phelan 2019; 8-9).
} 
order for the two individuals to become friends. However, this option neglects the case offered by Phelan himself, some work colleagues who are well-described as friends might eat lunch together once or twice a week but rarely interact otherwise (Phelan 2019; 3). It might be the case that such individuals sit closer to one another than they otherwise would, laugh at jokes that are generally socially frowned upon, or accept casual cursing (some of Phelan's examples of normmanipulation). But there seems to be no reason to think that they would necessarily do this. They might well enjoy one another's company without their relationship involving any norms that go beyond those that customarily apply to work colleagues. On this interpretation of Phelan's account, however, norm-manipulation is necessary for friendship, and so this possibility is ruled out. This seems too demanding to be a plausible constraint on friendships. Given this interpretation, the account seems overly narrow: it fails to accommodate many conventional friendships where the friends do not manipulate the norms that apply to their social positions.

\section{Interpretation 2: Background Norm-Manipulation}

Turning to the alternative interpretation, we might instead take the background norms that are manipulated by friends to be those that apply to strangers within a given cultural context. That is, in order to avoid the over-demandingness of the above interpretation, we might instead suggest that it is the background norms that apply to any two strangers within a social context that must be manipulated in order to be friends. This seems to make space for the conventional friendships that were ruled out by the above interpretations. After all, they were not interacting according to the norms that apply to strangers, and thereby can be counted as friends.

However, I will argue that if the relevant norms are those that apply to strangers, the condition is overly permissive. We can collaboratively manipulate norms for many different reasons, and many of these seem like the wrong kinds of reason to count as friends. Phelan thus introduces the mutual positive disposition condition to mark out the kind of norm manipulation that is relevant for friendship. However, this undermines the significance of norm-manipulation itself, since in that case norm-manipulation is itself explained by mutual liking.

As noted above, there are many different social scripts for different contexts. In a workplace we act in one way; in a bar the norms are different. Many of these social scripts are ones that we collaboratively opt in to. However, we do not intuitively want to count all such cases of norm-manipulation as constituting friendships. For instance, two individuals might jointly decide that they would work well together as business partners, and this would require manipulating many significant social norms. For such individuals, detailed discussions of their finances, though usually taboo, might become perfectly acceptable. After all, they are business partners. It might also be acceptable for such individuals to make significant claims on one another's time and energy in order to make business decisions, even where such claims would otherwise be unacceptable. It seems that on this interpretation this relationship would thus meet the norm manipulation requirement that Phelan proposes: since the partnership was freely entered into, the resultant relationship would be the product of significant collaborative norm-manipulation. However, the decision to become business partners could have been a wholly strategic business plan. Each individual could have entered into it based on a clear-eyed assessment of the compatibility of the

\footnotetext{
${ }^{4}$ One might think that here Phelan could suggest that these are not full, deep, or true friendships. But this kind of conception of friendship as an ideal is rejected by him. Moreover, we seem to ordinarily refer to some such relationships as 'friendship', a consideration he takes to have significant weight.
} 
other's expertise and skills, without any personal interest or feelings on either side. If this were the case, we would not be at all tempted to think that the two business partners are friends. The fact that their relationship is governed by norms that have been collaboratively manipulated seems to tell us nothing at all about whether it is well-described as a friendship. On this interpretation, the account is thus overly permissive.

In order to avoid these problems, Phelan suggests that there may be a second, distinct condition for friendship, which together with the norm-manipulation condition suffices for friendship. This second condition is that friends must have a 'mutual positive disposition' towards one another (Phelan 2019; 11). In one sense, the business partners might fulfil this condition (assuming that they have freely jointly chosen to work together): they presumably have a positive disposition towards their collaborator's capacity to successfully manage the business tasks on which they collaborate. Since this would not resolve the over-permissiveness of the account, it is presumably not the kind of positive disposition that Phelan has in mind. Many of the kinds of positive disposition that one might have towards another person, that is, do not seem relevant to friendship (one might think someone is a skilful businessperson, or physically attractive, or has an excellent memory, and it is does not seem that these need be relevant to friendship). The kinds of positive disposition that do seem to be relevant to friendship are rather attitudes such as liking or caring about the other person. ${ }^{5}$

This further condition plausibly allows us to isolate the kind of norm-manipulation that marks out friendship and thus resolves the over-permissiveness of the account. But it also reintroduces exactly the kind of condition that Phelan was initially at pains to exclude. The introduction of the mutual positive disposition condition thus seems at odds with Phelan's own initial motivations. ${ }^{6}$ Moreover, in the next section I shall suggest that if significant collaborative norm-manipulations fails to distinguish friendship, and in order to do so we must introduce the idea of a mutual positive disposition, norm-manipulation itself may not be a basic or core feature of friendship.

\section{Norm-Manipulation and Explanatory Power}

Crucially, once Phelan introduces the mutual positive disposition condition, it renders the norm-manipulation condition obsolete, because it plausibly explains norms manipulation. I will argue that this suggests that norm manipulation - even if it is a feature of friendship - is not a basic feature of friendship, and that it is thus wrong to conclude that friendship consists in relationships involving norm manipulation. Moreover, I will suggest that this is consistent with our ordinary ways of explaining norm manipulation: we commonly explain norm manipulation by reference to friendship, but not vice versa.

Phelan regards the mutual positive disposition condition (which we have seen must be something closer to liking) as a second necessary condition for friendship which together with the norm-manipulation condition is sufficient for friendship. This was intended as a way of avoiding

\footnotetext{
${ }^{5}$ In fact these alone do not necessarily seem to mark out friendship, since in friendship it seems to matter not only that the two individuals like (or care about etc.) one another, but that they are at least to some extent aware of the other person's liking. However, I am not looking here to provide a complete account of this condition.

${ }^{6}$ These cases of irrelevant kinds of mutual positive disposition make it start to look as if the relevant kind of mutual positive disposition is something suspiciously close to love or care. Given Phelan's initial motivations, he must show that the required positive disposition is distinct from those attitudes (love, care) that he began by rejecting as inessential for thinking about friendship.
} 
the over-permissiveness of the background norm-manipulation account: not every instance of norm-manipulation is relevant for friendship. However, once introduced, the mutual liking condition seems to render the norm manipulation condition obsolete, because norm-manipulation seems relevant only as a result of mutual liking. That is, the significance of norm-manipulation seems wholly explained by the fact when it is of the relevant kind it stems from mutual liking. When two people manipulate norms by sharing deeply personal information about themselves for example, it seems plausible that this kind of norm manipulation is relevant to their being friends, unlike the business partners' norm manipulation, only because their doing so is explained by their liking or caring about one another.

This, then, suggests that norm manipulation may be a feature of friendship, and perhaps even a ubiquitous one. But the mere fact that a feature is ubiquitous does not suffice to show that it is usefully included in our account or definition of that thing. The fact that friends manipulate social norms does not by itself suggest that friendship consists in collaborative norm manipulation. Such a feature may be a consequence of friendship, rather than a basic feature of it, and in this case it is not well thought of as definitive of friendship. Once Phelan re-introduces the condition that friendship involves mutual liking, it seems that this condition explains the norm-manipulation that is significant for friendship. Mutual liking, that is, distinguishes the cases in which norm manipulation is relevant and those in which it is not. However, it also seems to render norm manipulation explanatorily insignificant regarding friendship: the norm-manipulation seemingly matters only as a manifestation of mutual liking or caring. Liking, rather than norm manipulation, thus seems to be doing the explanatory work in this account.

That norm-manipulation is not basic for friendship also seems to explain our everyday ways of talking about friendship and norm manipulation. In general, we seem to take friendship to explain why people manipulate certain norms, rather than to be explained by it. For example, it seems natural to say that two people hug or otherwise manipulate norms concerning personal space 'because they're friends', whereas it would seem strange to say that two people are friends 'because they hug'. Similarly, the fact that someone is my friend seems like a good explanation of why I ask them for advice when I need it, whereas it would seem odd to say that we're friends because I ask them for advice. ${ }^{7}$ If norm-manipulation were basic to friendship, this would stand in need of explanation, because it would seem that the latter direction of explanation should make sense. Having seen that the norm manipulation involved in friendship seems relevant only because it arises from mutual liking, and that friendship seems to explain norm manipulation rather than vice versa, there is reason to think that Phelan has not offered an account of friendship that is a genuine alternative to the standard focus on deep relationships based on mutual caring.

\footnotetext{
${ }^{7}$ Norm manipulation might nonetheless play a causal role in forming friendships: if two people discuss something deeply personal, it is likely to be an opportunity to determine how aligned they are in terms of their worldview and temperament. It is also likely to reveal important things about each that the other might find appealing or attractive, and create a sense of intimacy. However, one can agree with all this and yet resist Phelan's claim that friendship consists in such norm manipulation.
} 


\section{Work Cited}

Annis, D (1987) “The Meaning, Value and Duties of Friendship” American Philosophical Quarterly, Vol.24 (4), pp.349-356

Helm, B (2010) Love, Friendship, and the Self: Intimacy, Identification, and the Social Nature of Persons (Oxford: Oxford University Press)

Nehamas, A (2010) “The Good of Friendship" Proceedings of the Aristotelian Society, Vol.110, pp.267-294

Phelan, M (2019), “Rethinking Friendship” Inquiry

Thomas, L (1987) “Friendship”, Synthese Vol.72(2), pp.217-236 